\title{
Altered DMN functional connectivity and regional homogeneity in partial epilepsy patients: a seventy cases study
}

\author{
Chong-Yu Hu${ }^{1}$, Xiaoping Gao ${ }^{1}$, Lili Long ${ }^{2}$, Xiaoyan Long ${ }^{2}$, Chaorong Liu², Yayu \\ Chen ${ }^{2}$, Yuanyuan Xie' ${ }^{2}$, Chujuan Liu ${ }^{3}$, Bo Xiao ${ }^{2}$ and Zhe-Yu Hu${ }^{4}$ \\ ${ }^{1}$ Department of Neurology, Hunan Provincial People's Hospital, Changsha, Hunan, China \\ ${ }^{2}$ Department of Neurology, Xiangya Hospital of Central South University, Changsha, Hunan, China \\ ${ }^{3}$ Department of Rehabilitation, Hunan Provincial People's Hospital, Changsha, Hunan, China \\ ${ }^{4}$ Department of Clinical Research and Teaching, The First Hospital of Changsha, Changsha, Hunan, China \\ Correspondence to: Bo Xiao, email: xiaobo_xy@126.com \\ Zhe-Yu Hu, email: zheyuhu@yahoo.com
}

Keywords: partial epilepsy, regional homogeneity, default mode network, resting state, BOLD-fMRI

Received: March 02, $2017 \quad$ Accepted: August 06, $2017 \quad$ Published: August 28, 2017

Copyright: Hu et al. This is an open-access article distributed under the terms of the Creative Commons Attribution License 3.0 (CC BY 3.0), which permits unrestricted use, distribution, and reproduction in any medium, provided the original author and source are credited.

\section{ABSTRACT}

Purpose: Clinically diagnosed partial epilepsy is hard to be functionally diagnosed by regular electroencephalograph (EEG) and conventional magnetic resonance imaging (MRI). By collecting transient brain regional signals, blood oxygenation level-dependent (BOLD) function MRI (BOLD-fMRI) can provide brain function change information with high accuracy. By using resting state BOLD-fMRI technique, we aim to investigate the changes of brain function in partial epilepsy patients.

Methods: BOLD-fMRI scanning was performed in $\mathbf{7 0}$ partial epilepsy and $\mathbf{7 0}$ healthy people. BOLD-fMRI data was analyzed by using the Regional Homogeneity (ReHo) method and functional connectivity of Default Mode Network (DMN) methods. The abnormal brain functional connectivity in partial epilepsy patients was detected by Statistical Parametric Mapping 8 (SPM8) analysis.

Results: Compared to healthy group, epilepsy patients showed significant decreased ReHo in left inferior parietal lobule/pre- and post-central gyrus, right thalamus/paracentral lobule/Cerebellum anterior and posterior Lobe, bilateral insula. The DMN functional connectivity regions decreased significantly in right uncus, left Inferior parietal lobule, left supramarginal gyrus, left uncus, left parahippocampa gyrus, and left superior temporal gyrus, in epilepsy patients, compared to healthy controls.

Significance: This study clarified that both ReHo and functional connectivity of DMN decreased in partial epilepsy patients compared to healthy controls. While left inferior parietal lobule was detected in both ReHo and DMN, many other identified regions were different by using these two BOLD-fMRI techniques. We propose that both ReHo and DMN patterns in BOLD-fMRI may suggest networks responsible for partial epilepsy genesis or progression.

\section{INTRODUCTION}

Epilepsy is a global problem, and more than 60 million people are affected globally in 2011 [1-2]. It is one of the most common chronic serious neurologic diseases with a incidence rate of $0.4 \%-1 \%$ globally and $0.6 \%-1 \%$ in developing countries [3]. There are two versions of epilepsy categorization: the International League Against Epilepsy (ILEA) 1981 criteria and ILEA
2010 criteria. Currently, version 1981 is commonly used in clinical settings [4]. It is the foundation of 2010 classification and 2017 novel classification [5]. According to 1981 classification, partial epilepsy refers to focal or localization-related seizures with temporal focal spike and wave complex on the electroencephalogram (EEG), which differs from the generalized types of epilepsy [6]. Cognitive dysfunctions, including dysmnesia, language barrier and emotional disturbance, are common clinical 
manifestations [7-8]. In some of the partial epilepsy patients, conventional MRI can locate the abnormal brain lesion. However, in other part of the patients, the causes of epilepsy are complex and unclear. In this study, we investigated this group of patients whose conventional MRI have no abnormity.

While EEG excels in detecting the brain electric activities signals longitudinally, fMRI focus more on the spatial and functional changes. Based on the change of local oxygen consumption and blood flow in neuronal activation, BOLD-fMRI can detect the transient local signals. In resting state, specific brain region could be stimulated and generate more oxyhemoglobin which result in relatively lower deoxyhemoglobin level and increase T2* weighted phase signal [9]. Currently, simultaneous EEG and fMRI are used to localize the abnormal areas in patients' brain, providing useful information for presurgery assessment [10]. Task-related BOLD-fMRI is much more frequently used to locate the lesions in language, memory and sensorimotor regions [11-12], which are important in pre-surgery planning. Compared to task-related fMRI, the resting state fMRI based on resting state BOLD signal is easier and more consistent [13].

Raichle, et al. [14], define the baseline state of brain activity as the resting state (awake and lying quietly with eye closed). In resting state, the ratio of oxygen used by brain to oxygen delivered by flowing blood (OEF) is uniform in some brain regions. This network distributes separately but highly functional related, including precuneus $(\mathrm{PCu}) /$ posterior cingulated cortex (PCC), medial prefrontal cortex (MPFC), ventral anterior cingulated cortex (cAVV), hippocampus, gyrus, parietal lobe, temporal lobe, etc. This network is called the Default Mode Network (DMN) and it is responsible for the readiness and alertness to changes in the external and internal environment [15]. During human behaviors and brain activities, local deviations in the OEF in DMN could be captured by BOLD-fMRI. For example, in temporal lobe epilepsy (TLE) patients, the BOLD signal increased in mesial temporal lobe, but decreased in DMN during seizure interval [16-17].

Regional homogeneity (ReHo) is another vital technique in resting state fMRI analysis [18]. Unlike functional connectivity, ReHo analyzes the synchronization during time series in neuronal activity regions. Abnormal ReHo signal indicates a neuron connection disorder [19]. Using ReHo alone, Yang, et al., suggested positive correlations between ReHo values in precuneus/PCC and supplementary motor area (SMA). Combined with DMN, Zeng, et al, identified a decreased ReHo pattern in DMN, which might be responsible for impairment of cognitive function in mesial temporal lobe epilepsy and hippocampus sclerosis (mTLE-HS) patients [20-21].

In this study, we collected 70 patients and performed both DMN and ReHo analysis. Compared to healthy group, left inferior parietal lobule was detected in both
ReHo and DMN. But many other regions identified by ReHo and DMN were different. So, we proposed that by using these two BOLD-fMRI techniques, both ReHo and DMN may suggest network responsible for partial epilepsy genesis or progression.

\section{MATERIALS AND METHODS}

\section{Participants}

\section{Patients}

BOLD-fMRI data were gathered in 70 right-handed partial epilespy cases in Xiangya Hospital and Hunan Provincial People's Hospital between January 2010 and June 2014. Of all patients, 33 are females and 37 are males. The diagnosis of partial epilepsy was based on clinical symptom, EEG and MRI test. The inclusion criterion:

a. According to 1981 International League Against Epilepsy (ILEA) classification, partial epilepsy has seizure $\geq$ twice per year;

b. Age of 14 to 50 years old (in clinical setting, brain development in people with 14-year old has reached adult level, so epilepsy patients at 14 visit department of adult neurology);

c. Educational levels higher than primary school graduation;

d. No abnormality in routine conventional MRI

e. Exclude patients with other transient brain dysfunction, such as syncope, transient ischemia attack (TIA), hysteria, and migraine;

f. Exclude patients with serious physical or mental diseases, patients with alcohol or drug abuse, patients with long-term usage of drugs except anti-epileptic drugs, or patients with family history of mental illness.

\section{Health controls}

seventy right-handed healthy people whose age, gender and education levels were matched with recruited patients were served as control (Table 1). This group of healthy people has no systematic neurology disease or symptoms and no family history of neurology disease. Routine conventional MRI findings are normal.

This study was approved by the institutional review Board of Health and Family Planning Commission of Hunan Provincial People's Hospital. The informed consent was obtained from each participant.

\section{MRI data acquisition}

Each participant lay supine with eye closed and body still. Head was fixed by a matched hood. During scanning, participants should awake and be relaxed without thinking. Several strategies were used: 1) To ensure patient to be awake during scanning, every recruited patient was asked if he or she fell asleep. If his or her answer is YES, repeated scanning was performed. 2) To make fMRI 
output comparable, every subject was instructed to follow the same steps: supine, stay static, close eyes, not thinking, and not sleep. 3) The scanning time was about 7 minutes per patient, and during this period of time all patient and healthy controls should keep awake.

Images were acquired using 3.0T scanner (Siemens magnet on trio, A Tim System, German) in Hunan Provincial People's Hospital. We performed location image scanning first, and then T1 scanning with following parameters: $\mathrm{TR}=1900, \mathrm{TE}=3.4 \mathrm{~ms}$, fov $=24 \times 24 \mathrm{~cm}$, matrix $=256 \times 256$, thickness $/$ gap $=1 / 0.5 \mathrm{~mm}, 160$ volumes. In this study, we did not detect any abnormality in our participants. Then, we continued to use T1 as located image to perform resting-state fMRI. The echoplanar imaging sequence has the following parameters: $\mathrm{TR}=3000, \mathrm{TE}=30 \mathrm{~ms}$, fov $=24 \times 24 \mathrm{~cm}$, matrix $=64 \times$ 64 , flip angel $=90^{\circ}$, thickness $/$ gap $=3 / 1 \mathrm{~mm}, 36$ volumes, scanning time $=7 \mathrm{~min} 08 \mathrm{sec}$.

\section{Data pre-processing}

MatLab (The Math Works Inc. Natick, Massachusetts, USA), MRIconvert (http://cni.uoregon. edu/jolinda/MRIConvert/), SPM8 (Statistical Parametric Mapping, http://www.fil.ion.ucl.ac.uk/spm/), and RESTing state fMRI data analysis toolkit (REST, by Song Xiaowei, http://resting-fmri.sourceforge.net) were used to preprocess MRI data and perform statistic analysis. The detailed steps were described as follow:

1) Converting acquired MRI figures to .img format by using MRIconvert software. Because magnetic field need time to become steady and patient also need some time to accommodate environment, so the first five time points MRI figures were deleted. The next 135 time-points were analyzed.

2) To eliminate head motion-induced noise, SPM8 was then used to evaluate the linear shifting of head on $\mathrm{x}$, $\mathrm{y}, \mathrm{z}$ axis, and the rotation of head around $\mathrm{x}, \mathrm{y}, \mathrm{z}$ axis. Only patients with linear shifting distances less than $1 \mathrm{~cm}$ and rotation angles less than $1^{\circ}$ were recruited.

3) Because anatomic structures of subjects' brains were different from each other, so their brain fMRI figures need to be normalized spatially. All testing fMRI figures were normalized by using standard SPM8 EPI template. Figure data were re-sampled based on a volume of $3 \times 3 \times$ $3 \mathrm{~mm}^{3}$ as a unit.

4) To reduce spatial noise and increase signal-tonoise ratio (SNR), we used $8 \times 8 \times 8 \mathrm{~mm}^{3}$ FWHM (fullwidth at half maximum) to smooth fMRI figures and eliminate the bias caused by spatial normalization.

5) Resting State fMRI data analysis toolkit (REST) were applied to remove baseline shift and filter noisy waves ( $\mathrm{f}<0.01 \mathrm{~Hz}$ or $\mathrm{f}>0.08 \mathrm{~Hz}$ ). Low-frequent baseline shift $(\mathrm{f}<0.01 \mathrm{~Hz})$ was generated by the heat noise brought by coil preamplifier. High-frequent physiologic noise ( $\mathrm{f}>$ $0.08 \mathrm{~Hz}$ ) was generated by breathing and heart-beating, etc.

\section{Regional homogeneity analysis}

MatLab and RESTing state fMRI data analysis toolkit (REST), and MRIconvert were used to perform Regional Homogeneity (ReHo) analysis.

In $\mathrm{fMRI}$, brain active region was formed by multiple connecting voxels in space. ReHo assumes selected voxel to be transiently similar to adjacent voxel. These ReHo voxels exhibit the same changes at the same time sequence. Here, we calculated the Kendall's coefficient concordance $(\mathrm{KCC})$ to evaluate the similarity between the selected voxel and the adjacent voxel [18]. In Matlab, REST package was used to calculate $\mathrm{KCC}$ values.

$$
\mathrm{W}=\frac{\mathrm{a}\left(\mathrm{R}_{\mathrm{i}}\right)^{2}-\mathrm{n}(\mathrm{R})^{2}}{\frac{1}{12} \mathrm{~K}^{2}\left(\mathrm{n}^{3}-\mathrm{n}\right)} \text {, where } n=135
$$

$\mathrm{W}$ measures the $\mathrm{KKC}$ value for selected voxel, ranging from 0 to 1 . $\mathrm{Ri}$ is the sum of rank for time point $\mathrm{i}$. $\overline{\mathrm{R}}=(\mathrm{n}+1) \times \mathrm{K} / 2$, indicating the average value of Ri. $\mathrm{K}$ is the number of calculation units (the number of adjacent voxels to form a minimal ReHo calculation unit). Here, $\mathrm{K}=27$, one selected voxel and 26 adjacent voxels.

, where $n=135$

\section{Functional connectivity analysis}

PCC and $\mathrm{PCu}$ are two adjacent areas, mainly located in Brodmann 7 (BA7) and 31 (BA31) regions. Here, we focused on $\mathrm{BA} 31$ region because Raichle, et al., reported that the $\mathrm{DMN} \mathrm{PCC} / \mathrm{PCu}$ localizes in $\mathrm{BA} 31$ region [14]. We chose a seed zone (circle) with a radius of $10 \mathrm{~mm}$ from the $\mathrm{PCu}$ center at coordinate point $=(-12,-47,32)[22]$. MetLab software was used to calculate the correlation coefficient of the seed zone compared with the whole brain in patients and in healthy controls. We then used Fisher's r-to-z conversion to transfer the correlation coefficient (r) to z statistics:

$$
Z=\frac{1+\mathrm{r}}{1-\mathrm{r}}
$$

Then, based on normal assumption, z statistics were adopted in following statistic analysis. $T$-test was performed to compare the difference of correlation coefficient between patients and healthy controls. According to $t$-test statistic results, differences of DMN functional connectivity region between patients and healthy controls were detected By using RESTing state fMRI data analysis toolkit (REST).

\section{Statistic analysis}

Numeric variables were summarized as mean (standard deviation). Categorical variables were reported as counts (percentage). To compare continuous variables (age and psychological assessment) that appear to have symmetric distributions between the two groups, analysis 
of variance was employed. To compare categorical variables between two treatment group, chi-square test or fisher's exact $(n<5)$ were performed.

One-sample $t$-test was performed to analyze the functional connectivity ( $z$ values) in each group. Twosample $t$-test was performed to compare the ReHo (W values) and functional connectivity values for matched case and control groups. A cluster $>50$ voxels with $p$-value $<0.001$ was considered as significant. All statistical analyses were conducted using MatLab.

\section{RESULTS}

\section{Demographics}

Of the 70 patient cases included for analysis, 8 cases were removed because of ineligible MRI quality. Among the remaining 62 cases, 30 were females and 32 were males. Age $(27.9 \pm 8.3 \mathrm{yr})$ ranged from $14 \mathrm{yr}$ to $48 \mathrm{yr}$. Of 70 controls, 6 cases were removed due to head motion or data quality reasons. Among the remaining 64 healthy controls, 29 were females and 35 were males. Age $=29.1 \pm$ 7.5 , ranged from 15 to 45 . As demonstrated in Table 1, no significant age, gender or educational disparity could be detected between case and control group.

Compared to healthy controls, the numerical depth, language fluency test (accuracy number) and logic memory in patients were significantly lower $(p<0.001)$. SDS score and SAS score in epilepsy patients were much higher than control group (Supplementary Table 1).

\section{DNM functional connectivity comparison in epilepsy patients and healthy controls}

By using resting state BOLD-fMRI scan and the functional connectivity analysis, we analyzed DMN regions in partial epilepsy patients and healthy controls. In healthy controls, the DMN functional connectivity regions covered left Pcu /PCC and angular gyrus, cingulated gyrus in epilepsy patients (Figure 1A). In healthy controls, the $\mathrm{DMN}$ functional connectivity regions included $\mathrm{Pcu} / \mathrm{PCC}$, right angular gyrus, bilateral medial frontal lobes and temporal lobes in healthy controls (Figure 1B). These findings further verified the existence of DMN at resting status. The DMN functional connectivity region in partial epilepsy patients was much smaller than the size of DMN functional connectivity regions in healthy controls. Detailed information was demonstrated in Supplementary Tables 2 and 3 .

In this study, seed zone was chosen (in DMN region) with a radius of $10 \mathrm{~mm}$ from the $\mathrm{PCu}$ center at coordinate point $=(-12,-47,32)$. MetLab software was used to calculate the correlation coefficient of the seed zone compared with the whole brain in patients and in healthy controls. $T$-test was performed to compare the difference of correlation coefficient between patients and healthy controls. Compared to healthy controls, the functional connectivity regions decreased significantly in epilepsy patients. Figure 2 showed the brain regions with decreased DMN functional connectivity in epilepsy patients, including right uncus, left Inferior parietal lobule, left supramarginal gyrus, left uncus, left parahippocampa gyrus, and left superior temporal gyrus. No increased DMN functional connectivity was observed in epilepsy patients.

Detailed information was demonstrated in Table 2. Supplementary Figure 1 showed the 3-dimension projection images for decreased DMN functional connectivity regions in epilepsy patients, compared with healthy controls. Supplementary Figure 3 demonstrated the decreased functional connectivity in projection map on axial brain image in epilepsy patients. In epilepsy patients, functional connectivity-decreased regions partly located in DMN regions, such as parietal lobe and temporal lobe. Other part of functional connectivity-decreased regions located outside of DMN regions.

In this study, all epilepsy patients had conscious disorders. For these patients, DMN was unable to maintain wakefulness and perform surveillance and selfexamination for internal and external environment. So, we supposed that the decreased DMN functional connectivity could be the mechanism for conscious disorders in these patients.

\section{Comparison of ReHo in epilepsy patients and healthy controls}

By using resting state BOLD-fMRI scan and ReHo analysis, no increased ReHo region was detected in this study. Instead, we found that the decreased ReHo regions covered left inferior parietal lobule/pre- and post-central gyrus, right thalamus/paracentral lobule/cerebellum anteriorand posterior Lobe, bilateral insula in epilepsy patients, compared to healthy controls (Figure 3). Detailed information for the decreased ReHo regions in epilepsy patients was described in Table 3. Supplementary Figure 2 showed the 3-dimension projection images for decreased ReHo regions in epilepsy patients, compared with healthy controls. Figure 3 showed the sagitta, coronal and axisal plane of the 3-D projection of ReHo map.

\section{DISCUSSION}

At resting state, synchronous low frequency fluctuations (LFFs) exist in multiple related brain regions. So, when pathological changes occur in some of these regions, we can detect abnormal LFFs. Functional connectivity and ReHo are two primary techniques at resting state for pathological brain region analysis. In this study, we performed these two analyses and found that compared to healthy group, epilepsy patients showed significant decreased ReHo in left inferior parietal 
Table 1: Demographics for patients $(n=62)$ and healthy controls $(n=64)$

\begin{tabular}{|c|c|c|c|c|}
\hline \multirow[b]{2}{*}{ variables } & \multirow[b]{2}{*}{ Levels } & \multicolumn{2}{|c|}{ Participants } & \multirow[b]{2}{*}{$p$-value } \\
\hline & & Patients $(n=62)$ & Controls $(n=70)$ & \\
\hline Age & & $27.9 \pm 8.3$ & $29.1 \pm 7.5$ & $0.39 * *$ \\
\hline \multirow[t]{2}{*}{ Sex } & Male & $32(51.6 \%)$ & $35(54.7 \%)$ & $0.73 \dagger$ \\
\hline & Female & $30(48.4 \%)$ & $29(45.3 \%)$ & \\
\hline \multirow[t]{3}{*}{ Education level } & Low education level & $5(8.06 \%)$ & $7(10.94 \%)$ & $0.80 \ddagger$ \\
\hline & High School or $*$ TSS & $30(48.4 \%)$ & $28(43.2 \%)$ & \\
\hline & College or above & $27(43.6 \%)$ & $29(45.3 \%)$ & \\
\hline
\end{tabular}

Note: $*$ TSS is technique secondary school. $* * t$-test was performed to calculate $p$-value for age at diagnosis; $\uparrow$ for variable sex, chi-square test was performed; + for variable educational level, low education level includes no education, primary school education and junior school education. Chi-square test was performaed to evaluate the association of education level with partial epilepsy.

lobule/pre- and post-central gyrus, right thalamus/ paracentral lobule/Cerebellum anterior and posterior Lobe, bilateral insula. The DMN functional connectivity regions decreased significantly in right uncus, left Inferior parietal lobule, left supramarginal gyrus, left uncus, left parahippocampa gyrus, and left superior temporal gyrus, in epilepsy patients, compared to healthy controls. No increased ReHo or DMN regions were identified in epilepsy patients.

In some studies, decreased ReHo was found to locate in regions of the DMN in epilepsy patients, such as children with new-onset drug-naïve benign epilepsy with centrotemporal spike (BECTS) and mTLE [20-21]. Here, we found that the decrease ReHo region, left inferior parietal lobule, localized in decreased DMN region.

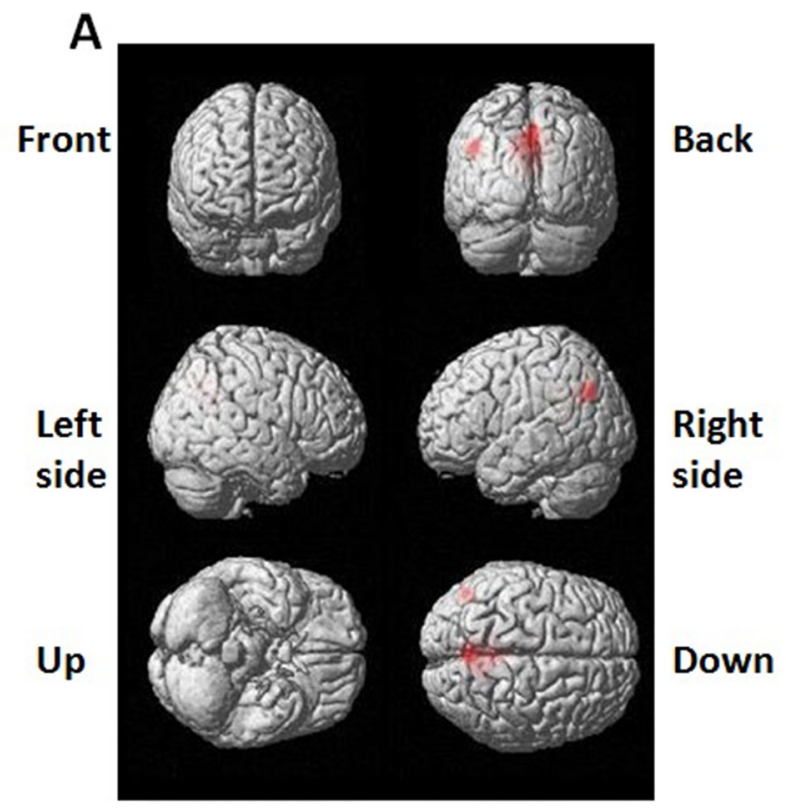

However, we still found many other identified regions to be different between ReHo and DMN patterns. Therefore, we proposed that ReHo and DMN patterns in BOLDfMRI might suggest partial epilepsy genesis, progression or other implications. For example, DMN alteration in TLE may be related to GABAergic and glutamatergic dysfunction [23], suggesting a implications of DMN in TLE pathophysiology and treatment surveillance [16]. Moreover, researchers implied that the decreased DMN connectivity might be diverse among different seizure types [24]. Here, due to the lack of evidence from EEG and MRI, we could not identify the seizure-related pathological loci in the tested 70 partial epilepsy patients. So, by using DMN/BOLD-fMRI, we were able identify possible DMN changes in all tested partial epilepsy patients.

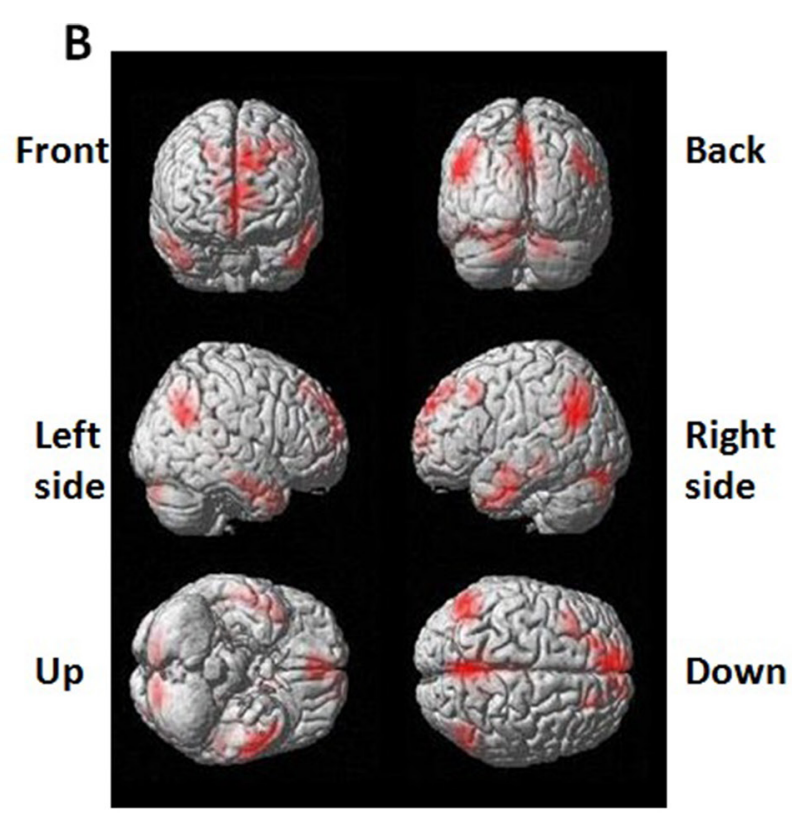

Figure 1: 3-dimensional projection images for DMN functional connectivity regions in (A) epilepsy patients and (B) healthy control . Red color indicates DMN regions. 
Table 2: Brain regions with decreased DMN functional connectivity in epilepsy patients

\begin{tabular}{|c|c|c|c|c|c|c|c|c|}
\hline \multirow[b]{2}{*}{ Pixel size } & \multirow[b]{2}{*}{$\mathbf{L} / \mathbf{R}$} & \multirow[b]{2}{*}{ Brain region } & \multirow[b]{2}{*}{ T statistics } & \multicolumn{5}{|c|}{ Coordinate } \\
\hline & & & & Z statistics & $\mathbf{x}$ & $\mathbf{y}$ & $\mathbf{z}$ & BA region \\
\hline 135 & $\mathrm{R}$ & Uncus & 4.10 & 3.67 & 33 & -15 & -36 & 20 \\
\hline \multirow[t]{2}{*}{66} & $\mathrm{~L}$ & Inferior Parietal Lobule & 4.95 & 4.35 & -57 & -39 & 48 & - \\
\hline & $\mathrm{L}$ & SupramarginaGyrus & 4.37 & 3.87 & -63 & -48 & 33 & 40 \\
\hline \multirow[t]{2}{*}{107} & $\mathrm{~L}$ & Uncus & 4.71 & 4.10 & -24 & 6 & -36 & - \\
\hline & $\mathrm{L}$ & ParahippocampaGyrus & 4.18 & 3.72 & -30 & -6 & -21 & - \\
\hline 60 & $\mathrm{~L}$ & Superior Temporal Gyrus & 3.89 & 3.45 & -48 & -39 & 6 & - \\
\hline
\end{tabular}

A
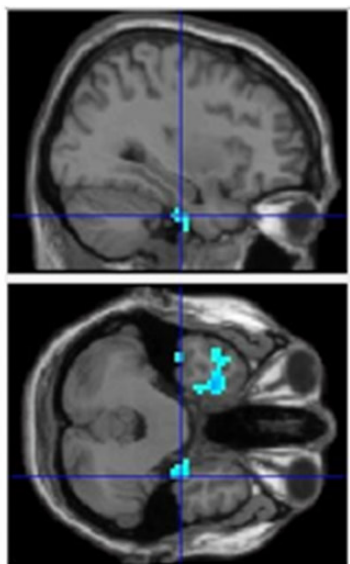

C
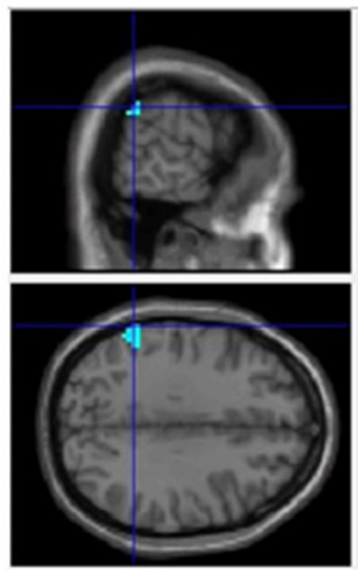

E
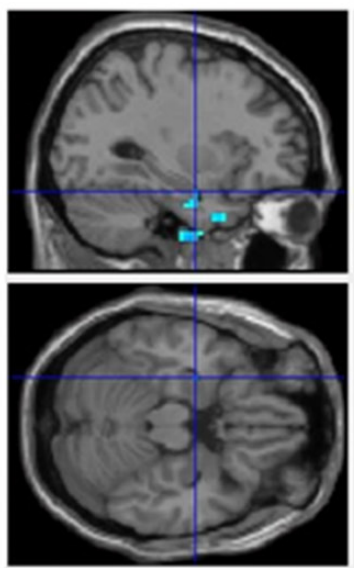
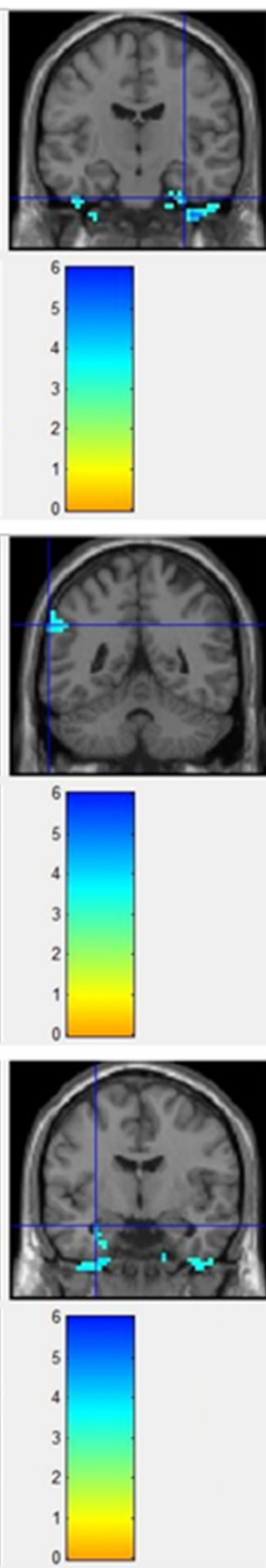

B
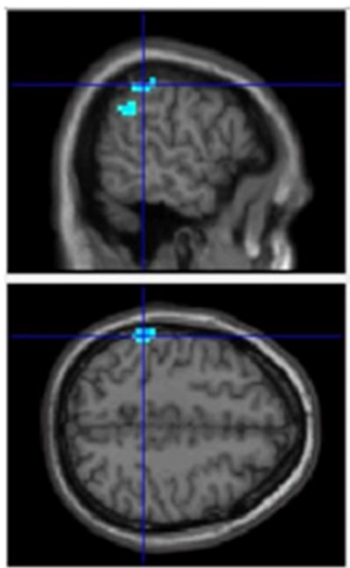

D
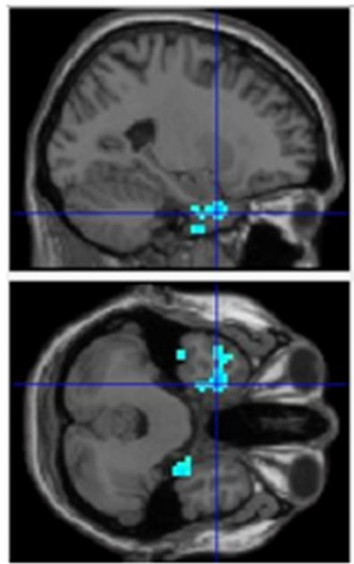

$\mathbf{F}$
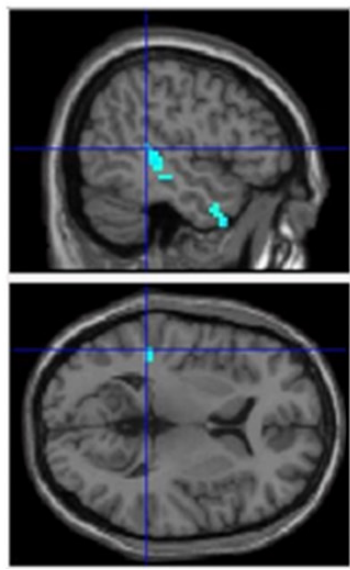
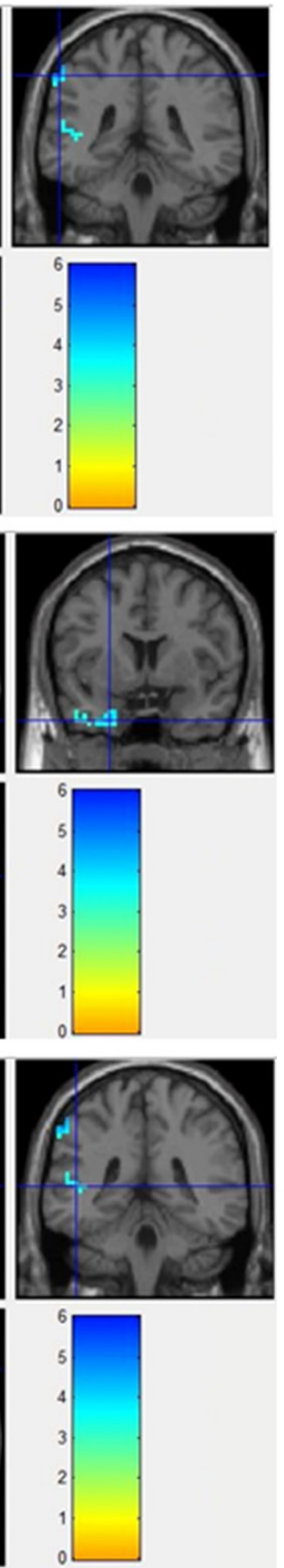

Figure 2: Brain regions with decreased DMN functional connectivity in epilepsy patients. (A) Right Uncus; (B) Left Inferior Parietal Lobule; (C) Left SupramarginalGyrus; (D) Left Uncus; (E) Left ParahippocampaGyrus; (F) Left Superior Temporal Gyrus 
Table 3: Brain regions with decreased ReHo in partial epilepsy patients

\begin{tabular}{|c|c|c|c|c|c|c|c|c|}
\hline \multirow[b]{2}{*}{ Pixel size } & \multirow[b]{2}{*}{$\mathbf{L} / \mathbf{R}$} & \multirow[b]{2}{*}{ Brain region } & \multirow[b]{2}{*}{ T statistics } & \multicolumn{5}{|c|}{ Coordinate } \\
\hline & & & & $\mathrm{Z}$ statistics & $\mathbf{x}$ & $\mathbf{y}$ & $\mathbf{Z}$ & BA region \\
\hline \multirow[t]{3}{*}{189} & $\mathrm{~L}$ & inferior parietal lobule & 7.36 & 5.23 & -39 & -48 & 57 & - \\
\hline & $\mathrm{L}$ & postcentralgyrus & 6.34 & 4.71 & -45 & -36 & 54 & 40 \\
\hline & $\mathrm{L}$ & precentralgyrus & 5.54 & 4.19 & -30 & -27 & 69 & 4 \\
\hline 558 & $\mathrm{~L}$ & insula & 6.41 & 5.07 & -30 & -24 & 15 & 13 \\
\hline \multirow[t]{3}{*}{302} & $\mathrm{R}$ & thalamus & 6.23 & 4.81 & 18 & -30 & 0 & - \\
\hline & $\mathrm{R}$ & insula & 6.09 & 4.51 & 36 & -21 & 15 & 13 \\
\hline & $\mathrm{R}$ & insula & 5.88 & 4.38 & 42 & -24 & -3 & 13 \\
\hline \multirow[t]{3}{*}{335} & $\mathrm{R}$ & Cerebellum Posterior Lobe & 6.45 & 4.72 & 12 & -72 & -27 & - \\
\hline & $\mathrm{R}$ & Cerebellum Anterior Lobe & 6.30 & 4.62 & 0 & -45 & -21 & - \\
\hline & $\mathrm{R}$ & Cerebellum Anterior Lobe & 5.79 & 4.36 & 3 & -60 & -9 & - \\
\hline \multirow[t]{2}{*}{57} & $\mathrm{R}$ & Cerebellum Anterior Lobe & 5.64 & 4.28 & 24 & -45 & -36 & - \\
\hline & $\mathrm{R}$ & Cerebellum Anterior Lobe & 4.57 & 3.59 & 18 & -42 & -27 & - \\
\hline \multirow[t]{2}{*}{89} & $\mathrm{R}$ & precuneus & 5.39 & 4.12 & 3 & -51 & 57 & 7 \\
\hline & $\mathrm{R}$ & paracentral lobule & 4.42 & 3.54 & 3 & -39 & 63 & 4 \\
\hline
\end{tabular}

Currently, the application of ReHo analysis in partial epilepsy was limited. It is only used in children or primary epilepsy. For example, children with rolandic epilepsy (RE, also called BECTS) showed increased ReHo signals in the central, premotor and prefrontal region, and decreased ReHo signals in bilateral orbitofrontal cortex and temporal pore [25]. Untreated children absence epilepsy (CEA) showed increased ReHo in bilateral insula and decreased ReHo in DMN region [26]. In primarily generalized tonic-clonic seizures (GTCS), bilaterally and symmetrically altered ReHo was observed in the cortical and subcortical structures [27]. In this study, we focused on the ReHo alterations in adult partial epilepsy patients. Even we lack EEG and conventional MRI evidence for the pathologic regions in adult partial epilepsy patients; we could identify decreased ReHo in inferior parietal lobule, precentral and postcentral gyrus, thalamus, paracentral lobule, cerebellum anterior and posterior Lobe, and bilateral insula. The decreased ReHo signal in local neuron areas might be caused by the damages in neurology network and brain functions after seizure discharges. In this study, ReHo described the synchronization of local neuron activity in neuron network, while DNM reflected the concordance of separate functional connectivity brain region. When combining these two methods, we could evaluate the disease characters more comprehensively.

ReHo and DMN functional connectivity evaluate brain functions from two different aspects. While ReHo mainly describes the synchronization of regional neuron activity in neural network, DMN functional connectivity measures the synchronization among brain regions. The combination of these two technologies would provide a more complete evaluation of the neural network for epilepsy patients. In this study, epilepsy patients showed significant decreased $\mathrm{ReHo}$ in $\mathrm{PCu}$, parietal lobe, frontal lobe, insula, thalamus and cerebellum, which is caused by the impairment in coordination of neuron activity within above brain region. ReHo findings suggest abnormalities in individual neural nodes. On the other hand, functional connectivity uses $\mathrm{PCu}$ as seed and the connectivity regions decreased significantly in parietal lobe, temporal lobe and Limbic Area, indicating obstruction of connecting pathway between $\mathrm{PCu}$ and above brain regions. There, ReHo combined with functional connectivity can evaluate both the intra-nodal activity and the inter-nodal connection of entire neural network.

As for the clinical symptoms, epilepsy patients showed significant lower concentration level in numerical depth test than health controls (Supplementary Table 1). Numerical depth test is used to evaluate patients' concentration ability. Inferior parietal lobe is responsible for concentration and stimulus surveillance [28]. Epilepsy patients showed significantly decreased ReHo and functional connectivity in inferior parietal lobe, suggesting the dysfunction of inferior parietal lobe as the pathophysiology basis for attention drop in epilepsy patients. Moreover, decreased $\mathrm{ReHo}$ in $\mathrm{PCu}$ region suggests a dysfunction of $\mathrm{PCu}$, which might be responsible for recurrent conscious disturbance [29-32]. Decreased ReHo in precentral gyrus suggests a decrease of coordination in precentral gyrus neurons, which is the pathology basis for deviated mouth and limb convulsion in epilepsy patients. In addition, decreased ReHo in insula is related to dysfunction of insula and deficiency of patients' language fluency and logic memory [33-34]. Thalamus is another region with decreased ReHo and its dysfunction is related to deficient learning and memory activity [35-36].

Besides ReHo, region with decreased DMN functional connectivity is also related to clinical symptoms. Limbic Area is constituted of gyrus subcallosum, cingulated gyrus, parahippocampal gyrus, the deep surface of hippocampus 


\section{A}
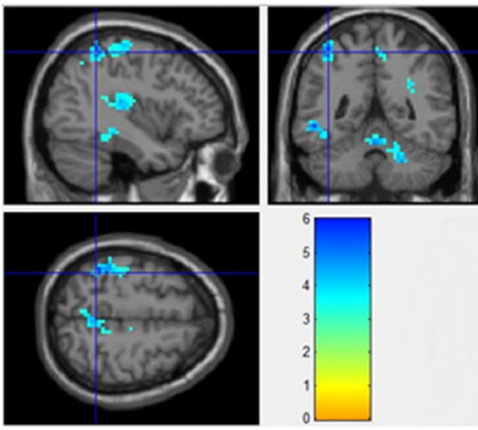

C
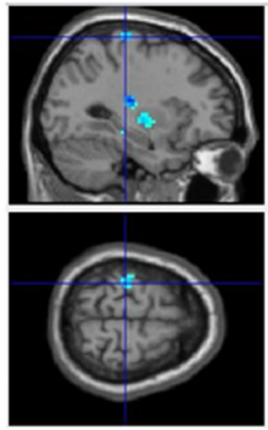

E
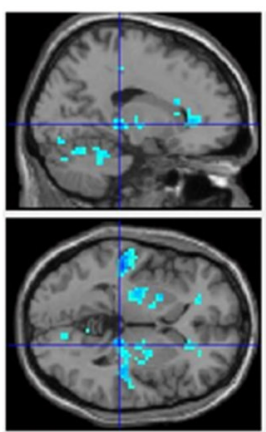

G
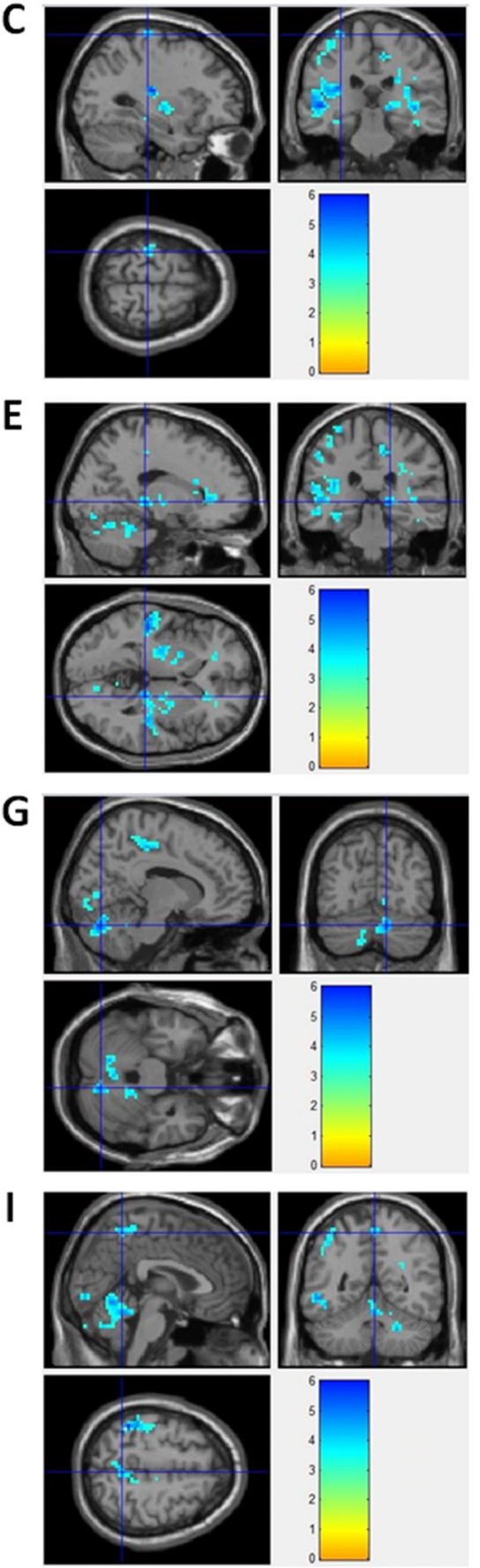

B
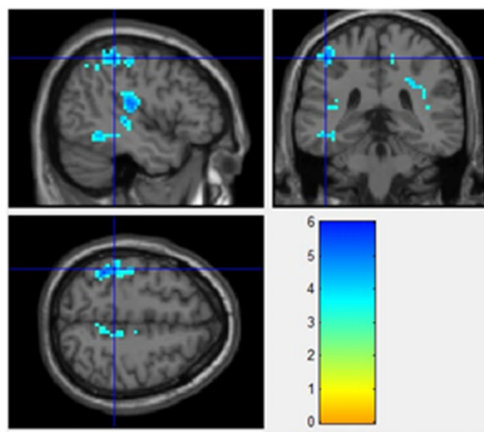

D
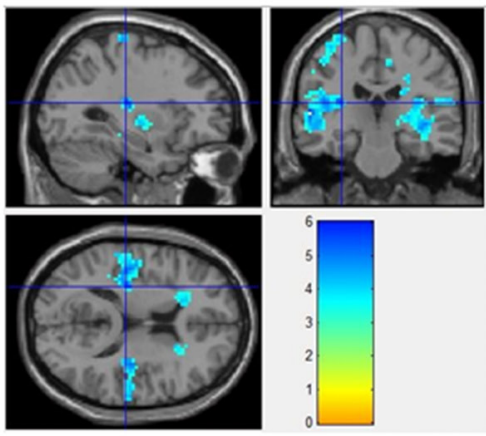

$\mathbf{F}$
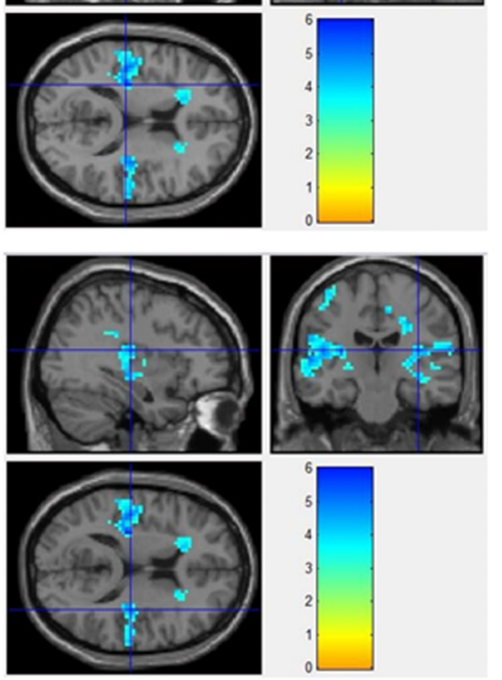

$\mathrm{H}$
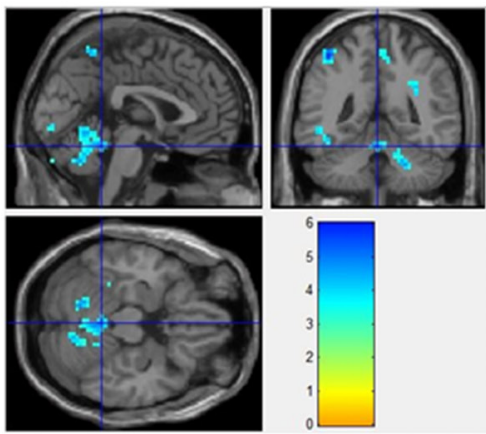

J
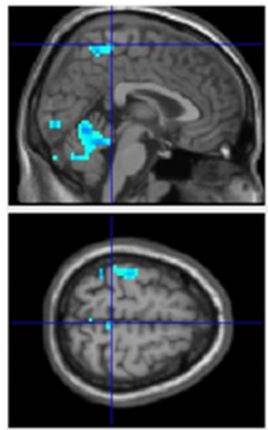

Figure 3: fMRI imagines for brain regions with decreased ReHo in epilepsy patients. (A) Left Inferior Parietal Lobule; (B) Left PostcentralGyrus; (C) Left PrecentralGyrus; (D) Left Insula; (E) Right Thalamus; (F) Right Insula; (G) Right Cerebellum Posterior Lobe; (H) Right Cerebellum Anterior Lobe; (I) Right Precuneus; (J) Right Paracentral Lobule. 
dentate gyrus, and related amygdale and hypothalamus. Limbic Area is an important part to regulate inner organs, feelings and emotion, and to participate into advanced knowledge activities, such as study and memory. Previous research had indicated that epileptic discharge of abnormal DMN region in patients might be conducted to Limbic Area, and lead to functional connectivity disorders between hippocampal gyrus and DMN region [37]. In this study, the functional connectivity between parahippocampal gyrus and DMN decreased significantly, suggesting a comprehensive inhibition of neural network. This abnormality is associated with decreased cognitive function, depression and anxieties in partial epilepsy patients. Moreover, decreased DMN functional connectivity of left superior temporal gyrus is associated with memory disorders.

Taken together, based on our findings in ReHo analysis and DMN functional connectivity of brain regions, we conclude that both the abnormal ReHo regions and decreased DMN functional connectivity regions are related to clinical symptoms in partial epilepsy patients. Further study will focus on the potential correlation between ReHo signal and DMN functional connectivities.

In study, patients with partial epilepsy were not categorized into specific epilepsy types, such as temporal lobe epilepsy. In future study, we can focuse on more specific type of partial epilepsy patients and try to optimize parameters in DMN and ReHo methods. Besides DMN and ReHo, other statistic methods could be developed in future in epilepsy diagnosis.

\section{Ethics approval and consent to participate}

This study was approved by the institutional review Board of Health and Family Planning Commission of Hunan Provincial People's Hospital. The informed consent was obtained from each participant.

\section{Author contributions}

Study concept and design: Chongyu Hu, Xiaoping Gao, Bo Xiao, Acquisition of data: Chongyu Hu, Analysis and interpretation of data: Chongyu Hu, Chujuan Liu, Yayu Chen, Yuanyuan Xie, Drafting of the manuscript and statistical analysis: Zheyu $\mathrm{Hu}$, Administrative, technical or material support: All authors.

\section{CONFLICTS OF INTEREST}

None.

\section{GRANT SUPPORT}

This study was supported by National Natural Science Foundation of China (No. NSFC- 81671300) and Xiangya Hospital Clinical Project (No. 2016L08).

\section{REFERENCES}

1. Perucca E, Covanis A, Dua T. Commentary: Epilepsy is a Global Problem. Epilepsia. 2014; 55:1326-8. https://doi. org/10.1111/epi.12725.

2. Thurman DJ, Beghi E, Begley CE, Berg AT, Buchhalter JR, Ding D, Hesdorffer DC, Hauser WA, Kazis L, Kobau R, Kroner B, Labiner D, Liow K, et al. Standards for epidemiologic studies and surveillance of epilepsy. Epilepsia. 2011; 52:2-26. https://doi.org/10.1111/j.15281167.2011.03121.x.

3. Brundtland GH. From the World Health Organization. Mental health: new understanding, new hope. JAMA. 2001; 286:2391. https://doi.org/jwh10001-1.

4. Unver O, Keskin SP, Uysal S, Unver A. The epidemiology of epilepsy in children: a report from a Turkish pediatric neurology clinic. J Child Neurol. 2015; 30:698-702. https:// doi.org/0883073814539559.

5. Fisher RS, Cross JH, French JA, Higurashi N, Hirsch E, Jansen FE, Lagae L, Moshe SL, Peltola J, Roulet Perez E, Scheffer IE, Zuberi SM. Operational classification of seizure types by the International League Against Epilepsy: Position Paper of the ILAE Commission for Classification and Terminology. Epilepsia. 2017; 58:522-30. https://doi. org/10.1111/epi.13670.

6. Schomer DL. Current concepts in neurology. Partial epilepsy. N Engl J Med. 1983; 309:536-9. https:/doi. org/10.1056/NEJM198309013090906.

7. Sveller C, Briellmann RS, Saling MM, Lillywhite L, Abbott DF, Masterton RA, Jackson GD. Relationship between language lateralization and handedness in lefthemispheric partial epilepsy. Neurology. 2006; 67:1813-17. https://doi.org/67/10/1813.

8. Meador KJ. Cognitive outcomes and predictive factors in epilepsy. Neurology. 2002; 58:S21-26. https://doi. org/10.1212/WNL.58.8_suppl_5.S21.

9. Ogawa S, Lee TM, Kay AR, Tank DW. Brain magnetic resonance imaging with contrast dependent on blood oxygenation. Proc Natl Acad Sci USA. 1990; 87:9868-72.

10. Manganotti P, Formaggio E, Gasparini A, Cerini R, Bongiovanni LG, Storti SF, Mucelli RP, Fiaschi A, Avesani M. Continuous EEG-fMRI in patients with partial epilepsy and focal interictal slow-wave discharges on EEG. Magn Reson Imaging. 2008; 26:1089-100. https://doi.org/ S0730-725X(08)00107-0.

11. Woermann FG, Jokeit H, Luerding R, Freitag H, Schulz R, Guertler S, Okujava M, Wolf P, Tuxhorn I, Ebner A. Language lateralization by Wada test and fMRI in 100 patients with epilepsy. Neurology. 2003; 61:699-701.

12. Guye M, Bettus G, Bartolomei F, Cozzone PJ. Graph theoretical analysis of structural and functional connectivity MRI in normal and pathological brain networks. MAGMA. 2010; 23:409-21. https://doi.org/10.1007/s10334-0100205-z. 
13. Qiao PF, Gao PY, Dai JP, Niu GM. Research progress on resting state fMRI of epilepsy. Brain Dev. 2012; 34:8-12. https://doi.org/S0387-7604(11)00005-2.

14. Raichle ME, MacLeod AM, Snyder AZ, Powers WJ, Gusnard DA, Shulman GL. A default mode of brain function. Proc Natl Acad Sci USA. 2001; 98:676-82.https:// doi.org/10.1073/pnas.98.2.676.

15. Harrison BJ, Pujol J, Lopez-Sola M, Hernandez-Ribas R, Deus J, Ortiz H, Soriano-Mas C, Yucel M, Pantelis C, Cardoner N. Consistency and functional specialization in the default mode brain network. Proc Natl Acad Sci USA. 2008; 105:9781-86. https://doi.org/0711791105.

16. Mohan A, Roberto AJ, Lorenzo A, Jones K, Carney MJ, Liogier-Weyback L, Hwang S, Lapidus KA. The Significance of the Default Mode Network (DMN) in Neurological and Neuropsychiatric Disorders: A Review. Yale J Biol Med. 2016; 89:49-57.

17. Pittau F, Grova C, Moeller F, Dubeau F, Gotman J. Patterns of altered functional connectivity in mesial temporal lobe epilepsy. Epilepsia. 2012; 53:1013-23. https://doi. org/10.1111/j.1528-1167.2012.03464.x.

18. Zang Y, Jiang T, Lu Y, He Y, Tian L. Regional homogeneity approach to fMRI data analysis. Neuroimage. 2004; 22:394-400. https://doi.org/10.1016/j.neuroimage.2003.12.

19. Thousands gather in Beijing for fourth women's conference. UN Chron. 1995; 32:66-7.

20. Zeng H, Pizarro R, Nair VA, La C, Prabhakaran V. Alterations in regional homogeneity of resting-state brain activity in mesial temporal lobe epilepsy. Epilepsia. 2013; 54:658-66. https://doi.org/10.1111/epi.12066.

21. Zeng H, Ramos CG, Nair VA, Hu Y, Liao J, La C, Chen L, Gan Y, Wen F, Hermann B, Prabhakaran V. Regional homogeneity (ReHo) changes in new onset versus chronic benign epilepsy of childhood with centrotemporal spikes (BECTS): A resting state fMRI study. Epilepsy Res. 2015; 116:79-85. https://doi.org/10.1016/j. eplepsyres.2015.06.017.

22. Greicius MD, Krasnow B, Reiss AL, Menon V. Functional connectivity in the resting brain: a network analysis of the default mode hypothesis. Proc Natl Acad Sci USA. 2003; 100:253-58. https://doi.org/10.1073/pnas.0135058100.

23. Nugent AC, Martinez A, D'Alfonso A, Zarate CA, Theodore WH. The relationship between glucose metabolism, resting-state fMRI BOLD signal, and GABAAbinding potential: a preliminary study in healthy subjects and those with temporal lobe epilepsy. J Cereb Blood Flow Metab. 2015; 35:583-91. https://doi.org/10.1038/ jcbfm.2014.228.

24. Widjaja E, Zamyadi M, Raybaud C, Snead OC, Smith ML. Impaired default mode network on resting-state FMRI in children with medically refractory epilepsy. AJNR Am J Neuroradiol. 2013; 34:552-57. https://doi.org/10.3174/ajnr. A3265.
25. Tang YL, Ji GJ, Yu Y, Wang J, Wang ZJ, Zang YF, Liao W, Ding MP. Altered regional homogeneity in rolandic epilepsy: a resting-state FMRI study. Biomed Res Int. 2014; 2014: 960395. https://doi.org/10.1155/2014/960395.

26. Yang T, Fang Z, Ren J, Xiao F, Li Q, Liu L, Lei D, Gong Q, Zhou D. Altered spontaneous activity in treatmentnaive childhood absence epilepsy revealed by Regional Homogeneity. J Neurol Sci. 2014; 340:58-62. http://dx.doi. org/10.1016/j.jns.2014.02.025.

27. Zhong Y, Lu G, Zhang Z, Jiao Q, Li K, Liu Y. Altered regional synchronization in epileptic patients with generalized tonicclonic seizures. Epilepsy Res. 2011; 97:83-91. https://doi. org/10.1016/j.eplepsyres.2011.07.007.

28. Singh-Curry V, Husain M. The functional role of the inferior parietal lobe in the dorsal and ventral stream dichotomy. Neuropsychologia. 2009; 47:1434-48. https:// doi.org/10.1016/j.neuropsychologia.2008.11.033.

29. Cavanna AE, Trimble MR. The precuneus: a review of its functional anatomy and behavioural correlates. Brain. 2006; 129:564-83. https://doi.org/10.1093/brain/awl004.

30. Vogt BA, Laureys S. Posterior cingulate, precuneal and retrosplenial cortices: cytology and components of the neural network correlates of consciousness. Prog Brain Res. 2005; 150:205-17. https://doi.org/10.1016/S00796123(05)50015-3.

31. Cavanna AE. The precuneus and consciousness. CNS Spectr. 2007; 12:545-52. https://doi.org/10.1017/ S1092852900021295.

32. Lundstrom BN, Petersson KM, Andersson J, Johansson M, Fransson P, Ingvar M. Isolating the retrieval of imagined pictures during episodic memory: activation of the left precuneus and left prefrontal cortex. Neuroimage. 2003; 20:1934-43. https://doi.org/S1053811903004671.

33. Augustine JR. Circuitry and functional aspects of the insular lobe in primates including humans. Brain Res Brain Res Rev. 1996; 22:229-44. https://doi.org/S0165017396000112.

34. Isnard J, Guenot M, Ostrowsky K, Sindou M, Mauguiere F. The role of the insular cortex in temporal lobe epilepsy. Ann Neurol. 2000; 48:614-23.

35. Hamandi K, Salek-Haddadi A, Laufs H, Liston A, Friston K, Fish DR, Duncan JS, Lemieux L. EEG-fMRI of idiopathic and secondarily generalized epilepsies. Neuroimage. 2006; 31:1700-10. https://doi.org/10.1016/j. neuroimage.2006.02.016.

36. Aghakhani Y, Bagshaw AP, Benar CG, Hawco C, Andermann F, Dubeau F, Gotman J. fMRI activation during spike and wave discharges in idiopathic generalized epilepsy. Brain. 2004; 127:1127-44. https://doi.org/10.1093/ brain/awh136.

37. Concha L, Beaulieu C, Gross DW. Bilateral limbic diffusion abnormalities in unilateral temporal lobe epilepsy. Ann Neurol. 2005; 57:188-96. https://doi.org/10.1002/ana.20334. 